\title{
Location dependent orientational structure and dynamics of Ethane in ZSM5
}

\author{
Siddharth Gautam ${ }^{1 *}$, Tingting Liu ${ }^{1}$, Sumant Patankar ${ }^{2}$, David Tomasko ${ }^{2}$ and David Cole ${ }^{1}$ \\ ${ }^{1}$ School of Earth Sciences, The Ohio State University, 275 Mendenhall Laboratory, 125 S Oval \\ Drive, Columbus, 43210, Ohio, United States of America \\ ${ }^{2}$ William G. Lowrie Department of Chemical and Biomolecular Engineering, The Ohio State \\ University, 151 W Woodruff Avenue, Columbus, 43210, Ohio, United States of America
}

\begin{abstract}
Orientational structure and dynamics of ethane confined in ZSM5 zeolite at four different loadings are reported. The effect of pore geometry on ethane is studied by isolating the contribution from ethane molecules in different locations, viz. straight channels, sinusoidal channels and their intersections. Orientational dynamics is found to be hindered in general and exhibits librational motion with the extant of hindrance being the greatest in sinusoidal channels. Librational motion becomes faster with an increase in loading. This counterintuitive finding is consistent with experiments reported elsewhere and is explained on the basis of a decreased orientational anisotropy at higher loadings.
\end{abstract}

Keywords: Rotation, Ethane, ZSM5, MD Simulation

*Corresponding author: gautam.25@osu.edu

Page 1 of 22

(C) 2016. This manuscript version is made available under the Elsevier user license

http://www.elsevier.com/open-access/userlicense/1.0/ 


\section{INTRODUCTION}

Behavior of fluids under confinement or at solid substrates is an interesting topic of research from the point of view of both applications and fundamental interests [1 -4$]$. Most of the properties of fluids are affected by confinement and exhibit deviations from the bulk. The phase behavior [5 - 7], structure [8 - 10] as well as dynamics [11 - 13] of molecules have been shown to be affected on confinement. Diffusive properties of hydrocarbons in zeolites is important in the roles they play in catalysis, separation and gas extraction industries. Orientational dynamics (rotation) of these hydrocarbons is an important component of these diffusive properties. While long range translational structure and dynamics get affected even by a mild confinement, where the dimensions of the confining space is several times the size of the confined molecules, the short range orientational structure and dynamics are affected only when the dimension of the confined space is comparable to the size of the confined molecule. For example, the rotational motion of water has been shown to remain mostly unaffected on confinement by the layered saponite structure with an interlayer spacing between 0.8 to $1.4 \mathrm{~nm}$, whereas translational motion is seen to be affected [14]. In a recent MD simulation study of water confined in $\mathrm{TiO}_{2}$ pores of different sizes $(1.3 \mathrm{~nm}$ to $5.1 \mathrm{~nm})$, water was found to exhibit two regimes of rotational motion with the faster rotational motion of water approaching the time scales observed in the bulk for larger pores [15]. Most hydrocarbons have been shown to exhibit isotropic rotation when confined to pores much larger than the hydrocarbon molecule studied. For example, the rotation of acetylene, propane, propylene and 1,3-butadiene have been found to be isotropic in Na-Y zeolite, with pore size $(1.2 \mathrm{~nm})$ more than twice the kinetic diameter of these molecules, both experimentally and when studied using computer simulations [16 - 20]. When the confining space is reduced further, as in ZSM5 with pore dimensions of about $0.55 \mathrm{~nm}$, the rotational 
motion of propylene is found to be hindered and exhibits librational motion [21]. A similar hindrance to isotropic rotation of ethane in Na-ZSM5 was observed in neutron scattering experiments [22]. Thus, ZSM5 is a good confining medium to study the effects of confinement on the orientational dynamics (rotation) of molecules. Moreover, having three different types of pore geometries (straight channels in the Y-direction, sinusoidal channels in the $\mathrm{X}-\mathrm{Z}$ plane and roughly spherical intersection of the two types of channels), there is an opportunity to study the role of pore geometry in this confinement effect by isolating the effects of confinement due to different pores. The results obtained here can thus be generalised to other zeolites with similar pore dimensions.

The librational motion of propylene in ZSM5 was found to completely trace the whole orientational space when the propylene molecules transit from one type of channel to the other [21]. Thus overall, there appeared to be no preference for a particular orientation. However, in a single channel the orientational space was restricted. Thus the behavior of the confined molecule in a given channel can be different from the overall behavior and so it is important to probe how the orientational structure and dynamics of the confined molecules differ in the pores with different geometries. Reducing the rotational degrees of freedom by choosing a dumbbell shaped molecule simplifies the system and thus makes a study of orientational structure and dynamics convenient. With this in mind we carried out an MD simulation study of ethane confined in ZSM5. The properties of confined ethane were studied for different loadings and in different pore geometries. The dependence of orientational motion on loading is found to be consistent with that observed in the neutron scattering experiments [22]. A decrease in the anisotropy in the orientational distribution of confined ethane molecules with increasing loadings explains the loading dependence of orientational motion. 


\section{SIMULATION DETAILS}

MD simulations reported here were carried out using the software DL_POLY [23]. The

calculations were performed using the Deep Carbon Observatory cluster at the Rensselaer Polytechnic Institute, Troy, USA. ZSM5 structure was modeled using the atomic positions reported by Konigsveld et al. [24]. The simulation cell consisted of $2 \times 2 \times 3$ unit cells of ZSM5 (silicalite-1) resulting in a simulation box with dimensions of $40.044 \AA$ × $39.798 \AA \times 40.149 \AA$. Ethane molecules were loaded in ZSM5 at four different loadings of $n=2,4,6$ and 8 molecules per unit cell (mpuc) leading to total number of ethane molecules of 24, 48, 72 and 96 respectively. Ethane molecules were modeled in the united atom formalism and consisted of two $\mathrm{CH}_{3}$ sites connected by a rigid bond of length $1.54 \AA$. TraPPE-UA [25] force field was used to model the interaction between ethane molecules and ZSM5 interacted with ethane molecules with a Lennard-Jones potential. The potential parameters for all these interactions were taken from the literature $[25,26]$. Following the TraPPE-UA convention, all interactions were truncated at a cut-off distance of $14 \AA$. All ZSM5 atoms were kept rigid throughout the simulation. A time step of $1 \mathrm{fs}$ was used. Following the fluctuations in temperature and total energy of the system, it was observed that the system attains equilibrium within $0.5 \mathrm{~ns}$. Therefore, an initial simulation of $0.5 \mathrm{~ns}$ was used for the equilibration of the system and trajectories were recorded for calculation of the thermodynamic properties from a simulation run of $1.5 \mathrm{~ns}$ that followed. All simulations were carried out at a temperature of $300 \mathrm{~K}$ in the NVT ensemble employing the Nose-Hoover thermostat. To isolate the properties of ethane in different pore types, the location of all the channels were determined from the ZSM5 structure. Figure 1 shows the structure of ZSM5 along with the locations of straight channels and three 
representative sinusoidal channels. We isolated three different types of pore spaces - straight channels, sinusoidal channels and the intersections. It should be noted however that the intersections form parts of both the straight as well as the sinusoidal channels. Thus, to avoid overlap, we refer to the space in the straight and sinusoidal channels other than the intersections when we talk about straight and sinusoidal channels respectively. Properties were calculated for ethane molecules that had their center of mass lying within these different pore types.
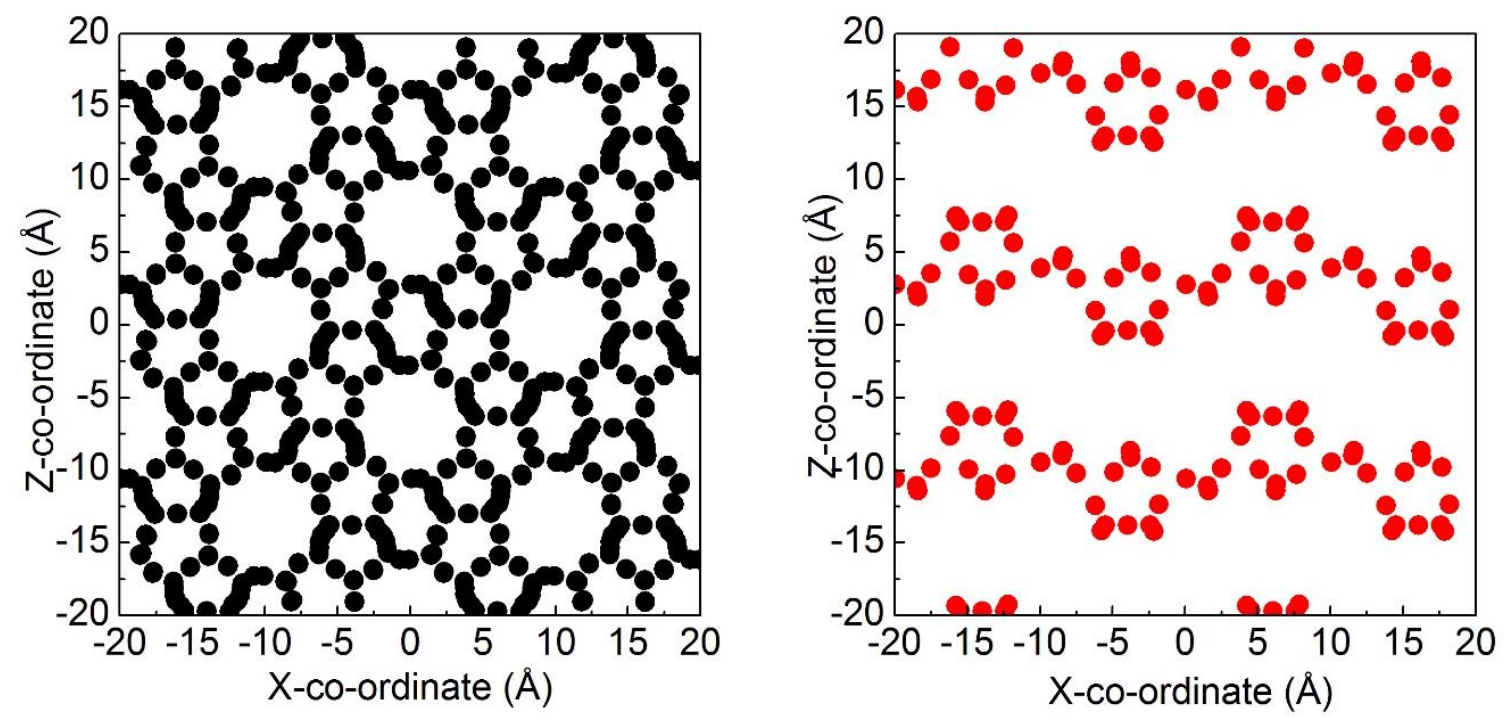

Figure 1. Structure of ZSM5. Left panel shows projection of all atoms in a $2 \times 2 \times 3$ unit cell on the $\mathrm{X}-\mathrm{Z}$ plane. Eight straight channels along the Y-direction can be seen lying completely in the unit cell. The right panel shows a cross-section of the cell in the $X-Z$ plane between $y=-17.0$ and -14.0 Å. Sinusoidal channels running mostly along the X-direction can be seen as empty space.

\section{RESULTS}

\section{A. Residence of ethane in different pore locations}


It is useful to know where the ethane molecules prefer to reside. To understand this, we calculated the residence autocorrelation functions for ethane in different pore types, $R_{i}$, defined as

$$
R_{i}(t)=\frac{\left\langle\theta_{i}(t) \cdot \theta_{i}(0)\right\rangle}{\left\langle\theta_{i}^{2}(0)\right\rangle}
$$

where $\theta_{i}$ is a Heaviside step function that takes the value of 1 if a molecule was in the pore type $i$ at time $t$ and all previous times and 0 otherwise. These functions give the probability of finding a molecule in a given pore type after a time $t$ given that the molecule was present in that pore type at time $t=0$.

Figure 2 shows the residence autocorrelation functions in different locations of ZSM5 for $n=8$ mpuc. Evidently, the confined ethane molecules have a propensity to remain localized at the intersections of the straight and sinusoidal channels the most. The $R_{i}(t)$ for the straight channels decay slightly faster as compared to that for the sinusoidal channels. This is probably because the simple geometry of these channels makes it easier for a typical ethane molecule to exit these channels as compared to the sinusoidal channels. We calculated the residence time of an ethane molecule in a given pore space by approximating the corresponding $R_{i}(t)$ functions by an exponential decay function. The decay constant of these functions represent the residence time of ethane in the pores. For the loading of 8 mpuc, residence times of 55, 18 and 20 ps were obtained for ethane molecules in intersections, straight channels and sinusoidal channels respectively. Similar trends in the residence times in different pores were obtained for the loadings of 6 and 4 mpuc, whereas at the lowest loading of 2 mpuc, the small number of molecules makes the distinction in residence times statistically difficult to resolve for different pores. 


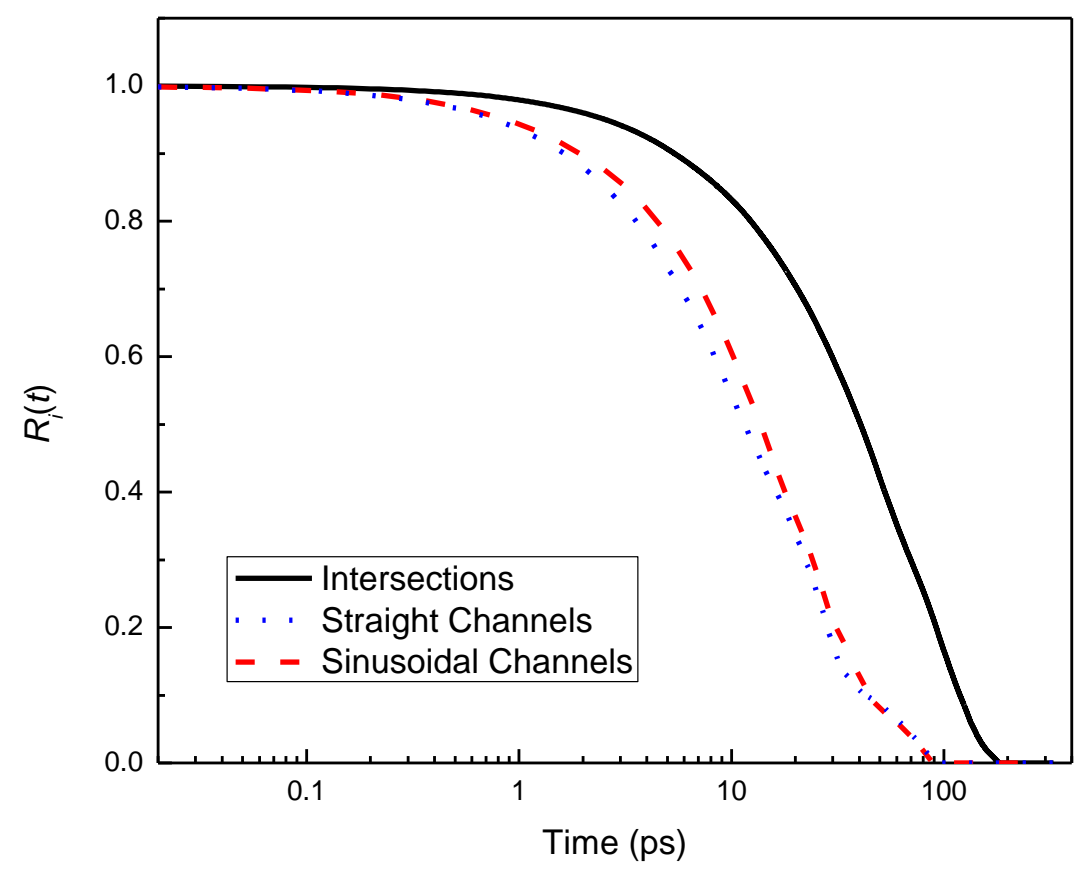

Figure 2. Residence Correlation Functions for ethane in different pore types at a loading of $n=8$ mpuc.

\section{B. Orientation of Ethane}

We calculated the orientational distribution function $g(\theta)$ of ethane molecules in ZSM5 by counting the number of molecule that have their molecular axis making an angle $\theta$ with a Cartesian axis at any given time. These orientational distribution functions with respect to the three Cartesian axes are shown in Figure 3 for the overall as well as the three pore types for $n=8$ mpuc. In case of an absence of a preferred orientation, this distribution would be isotropic. It is straightforward to show that this isotropic distribution would be proportional to $2 \pi r \sin \theta$ for a molecule with radius of gyration $r$. This isotropic distribution for ethane is also shown in Figure 3 as a reference along with the calculated distributions in ZSM5. 
Overall, in ZSM5, ethane molecules can be seen to exhibit preferential orientations resulting in a deviation from the isotropic behavior. A typical ethane molecule shows a preference to lie either close to parallel or perpendicular to the $\mathrm{X}$ and $\mathrm{Y}$ directions whereas mostly perpendicular to the $\mathrm{Z}$ direction, as is evident from the peaks around angles 25,90 and $155^{\circ}$. This is a direct consequence of the pore geometry. As can be seen from Figure 1, the straight channels are parallel to the $\mathrm{Y}$ direction and perpendicular to the $\mathrm{X}-\mathrm{Z}$ plane. The sinusoidal channels, however, are not perfectly parallel to $\mathrm{X}$-direction, lie mostly along $\mathrm{X}$-directions with a very small component along the Z-direction and are perpendicular to the Y-direction. Thus most of the ethane molecules would lie perpendicular to the $\mathrm{Z}$ direction and along either $\mathrm{X}$ or $\mathrm{Y}$ direction. The intersection of the sinusoidal and straight channels gives rise to a relatively larger pore space in the intersections which imposes a less severe restriction on the confined molecules. The orientation of a typical ethane molecule in the intersections is therefore closer to that expected for an isotropic distribution. In the straight and sinusoidal channels this orientational freedom is restricted and the ethane molecules show a strong preference in orienting parallel to $\mathrm{Y}$ and $\mathrm{X}$ directions respectively lying perpendicular to the $\mathrm{Z}$ direction in either case.

The deviation from the isotropic behavior facilitates quantifying the degree of anisotropy in orientational distribution by defining a parameter $\varphi$ as the root mean squared (rms) deviation from the isotropic behavior.

$$
\varphi=\sqrt{\frac{1}{N} \sum_{i}^{N}\left(g_{i s o}\left(\theta_{i}\right)-g_{z s m}\left(\theta_{i}\right)\right)^{2}}
$$

where $g$ is the orientational distribution function and the subscripts iso and zsm stand for the isotropic case and the observed case of ethane in ZSM5. The sum is taken over $N=1000$ different values of the angle $\theta_{i}$ between 0 and $180^{\circ}$ for which the distribution functions were calculated. 
The parameter $\varphi$ is shown in Figure 4 as a function of different loadings for different pore types. There is a tendency for the anisotropy to reduce on increase in loading. This is because at lower loadings, the ethane molecules take orientations that are energetically most favorable. As the loading is increased, the most favorable orientations already taken by the ethane molecules at lower loadings are no longer available and so the newly added ethane molecules have to take the next most favorable orientations. This increases the number of different orientations taken by the molecules, thus reducing anisotropy in the orientational distribution. The reduction in anisotropy in the orientational distribution with increase in loading is most prominent in the straight channels. This is because of the highly anisotropic environment in these channels to start with. The intersections fail to show a monotonic variation in the anisotropy with loading because of a very small anisotropy. The straight channels show stronger anisotropy along the Y-direction while the sinusoidal channels show stronger anisotropy along the $\mathrm{X}$-direction due to their alignment along these two directions. 

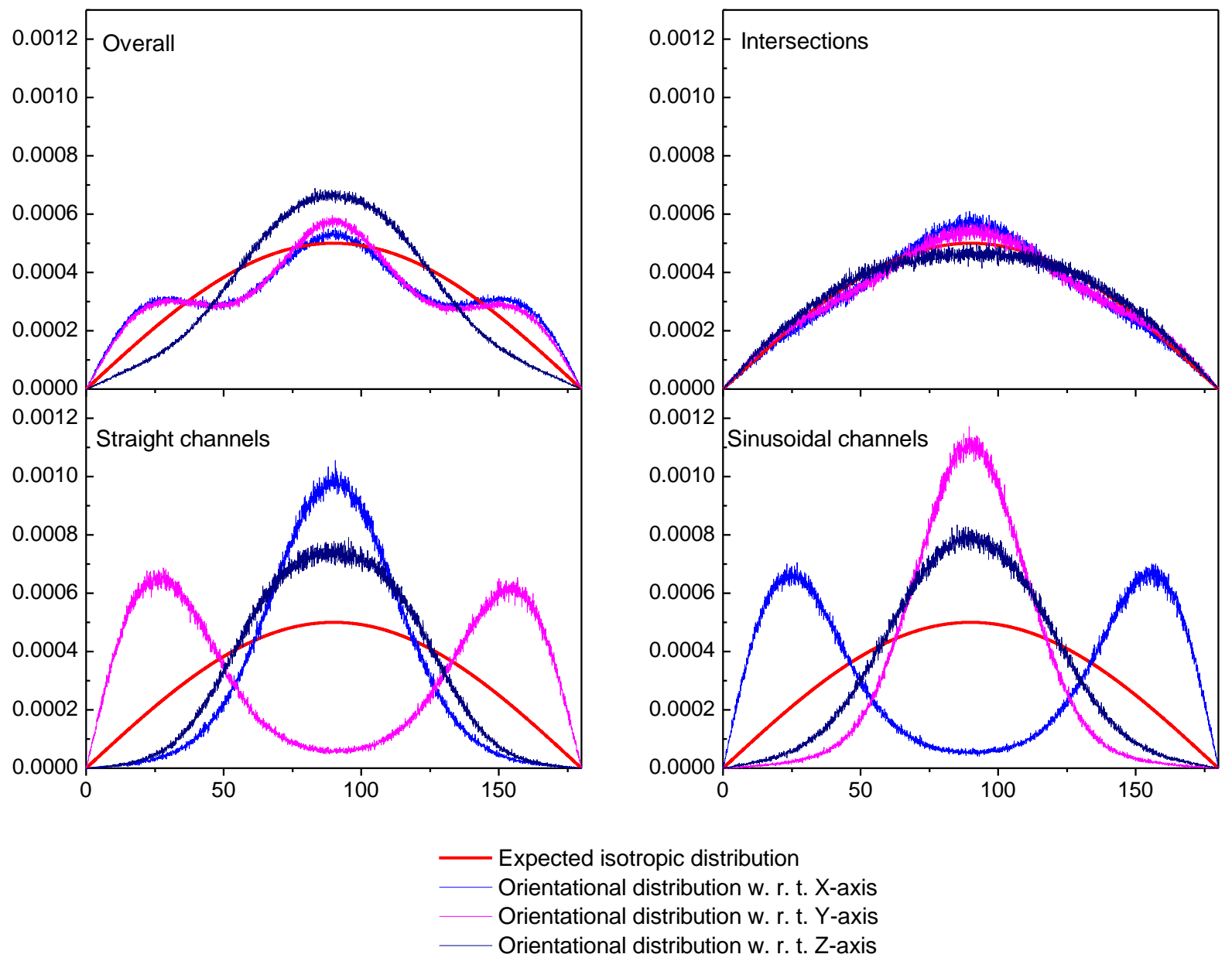

Figure 3 Orientation distribution of ethane in ZSM5 (top left), in intersections (top right), in straight channels (bottom left) and sinusoidal channels (bottom right). The orientational distribution expected for an ideal isotropic behavior is also shown as a reference. 


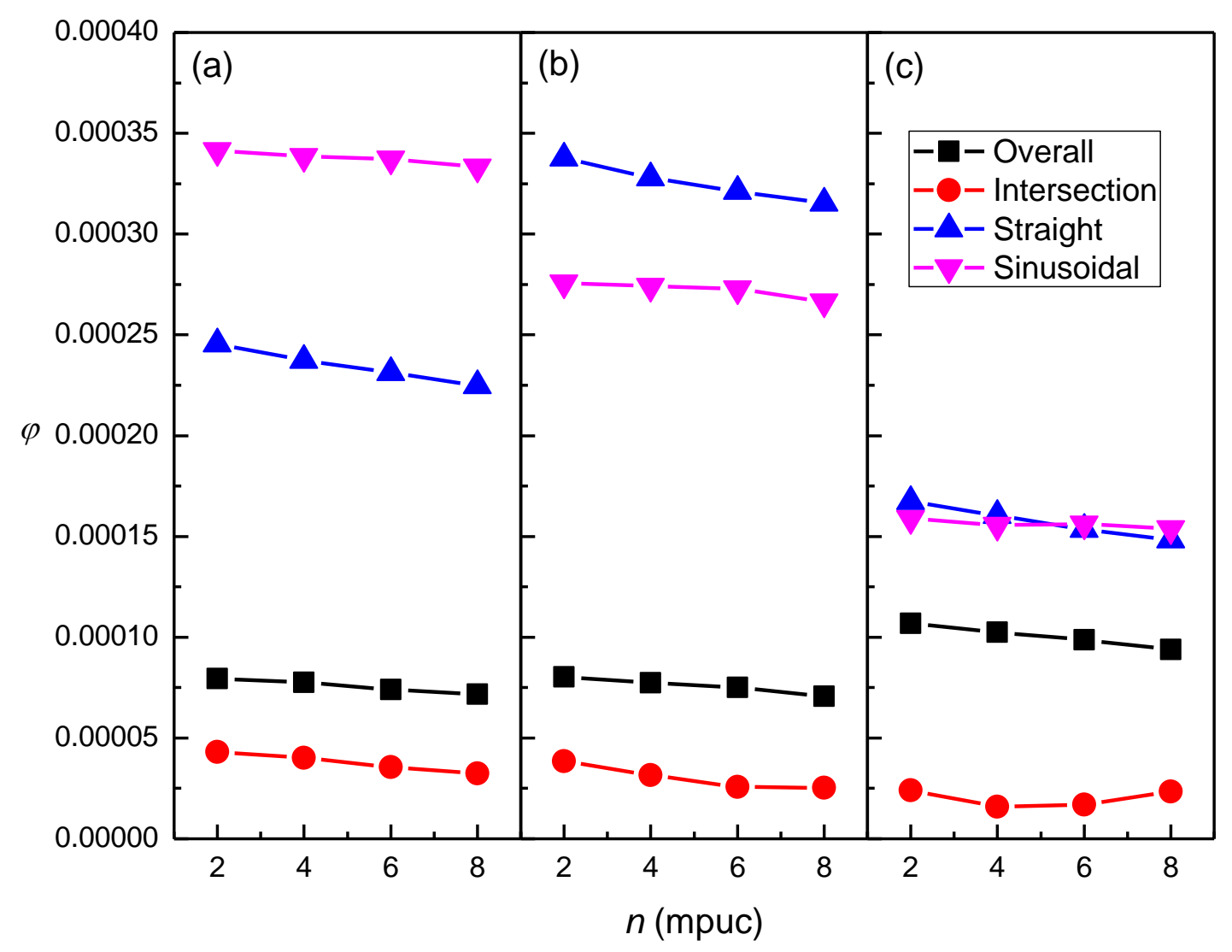

Figure 4 Anisotropy parameter along (a) X-axis, (b) Y-axis and (c) Z-axis as a function of ethane loading for different pore types.

\section{Dynamics}

Orientational dynamics (rotation) of ethane in ZSM5 can be studied by following the evolution of a $\mathrm{CH}_{3}$ site constituting the molecule in the Center of Mass frame of reference. As the molecular bond length has been kept fixed throughout the simulation, this would yield the orientational motion of ethane without any vibrational component. We use a unit vector $(\boldsymbol{u})$ attached to the molecular axis in the center of mass frame to study the orientational motion. This unit vector forms a unit dipole, the autocorrelation of which, can be used to obtain the time scale 
and the geometry of orientational motion. Specifically, one can calculate the orientational correlation functions (OCFs) of different orders as

$$
C_{l}(t)=\left\langle P_{l}[\boldsymbol{u}(t) \cdot \boldsymbol{u}(0)]\right\rangle
$$

where $P_{l}[x]$ is a Legendre polynomial of $x$ of order $l$.

The orientational correlation functions of different orders can be measured in experiments. $C_{l}(t)$ can be seen to represent the dipole correlation function that can be measured in a dielectric relaxation spectroscopy experiment. $\quad C_{2}(t)$ on the other hand can be obtained in an NMR measurement. In Figure 5 we show the overall OCFs for ethane in ZSM5 for different loadings. The loading dependence of the OCFs can be appreciated from the behavior of the first order OCF shown in the inset. The decay of the first order OCF gets faster at higher loadings.

In an unrestricted environment the OCFs are expected to exhibit exponential decay behavior. However in presence of severe restrictions imposed on the molecular rotation by the immediate environment of the molecule, a complete rotation is hindered and the molecule is left oscillating between different orientations. This type of hindered orientational motion of the molecule is referred to as librational motion. The troughs and peaks exhibited in the OCFs shown in Figure 5 are a signature of such librational motion. This has been observed for propylene in ZSM5 [21].

The time scales involved in the librational motion can be obtained by integrating the OCFs to give correlation times $\tau_{l}$.

$$
\tau_{l}=\int_{0}^{\infty} C_{l}(t) d t
$$

In practice, the OCFs decay to zero after some time and so the upper limit of integration can be reduced to a finite time. Once these functions have decayed to zero, at a given time, extending 
the upper limit of integration to longer times might have contributions from statistical fluctuations at large times. We found that $t=700 \mathrm{ps}$ was long enough for the OCFs for all loadings to decay to zero, but short enough to minimize spurious contribution to the integral due to statistical fluctuations. The correlation times were therefore evaluated according to Eq. 4 with the upper limit of $t=700 \mathrm{ps}$. The correlation times of different orders obtained for different loadings are shown in Figure 6. It can be seen that the overall rotation of ethane in ZSM5 gets faster on increase in loading.

The OCFs were calculated for molecules residing in different pore types. The first order OCFs calculated for intersections, straight channels and sinusoidal channels are shown in Figure 7 along with the overall OCFs for $n=8$ mpuc. The functions for different pores decay with a considerable difference in the decay rates. While the decay is slowest for the sinusoidal channels, the interesections exhibit the fastest decaying OCF. This is to be expected as the sinusoidal channels have a severely restricted and convoluted space and the intersections are larger and almost spherical. We should be cautious however while quantifying this difference. It should be noted that a typical ethane molecule would not remain in a given pore for more than the residence time in that pore and so the orientational correlations beyond the residence time of an ethane molecule in a particular pore are no longer meaningful. This means that the correlation times as obtained for the overall OCFs using Eq. 4 above cannot be obtained for different pores. However, we can talk about the difference in the behavior of OCFs in different pores up to 1 ps. This time is long enough for the differences to appear and at the same time short enough for the molecules to remain in the pore that they were in originally. It can be seen that the $C_{l}(t=1 \mathrm{ps})$ values for sinusoidal channels are the largest indicating a slower decaying OCF in this pore type and thereby implying a slower rotation. The intersections exhibit the lowest $C_{l}(t=1 \mathrm{ps})$ values 
indicating a faster rotation. As seen in Figure 5, the difference in the OCFs for different loadings gets prominent after $10 \mathrm{ps.} \mathrm{However} \mathrm{this} \mathrm{difference} \mathrm{exists} \mathrm{even} \mathrm{at} \mathrm{short} \mathrm{times} \mathrm{even} \mathrm{though} \mathrm{not} \mathrm{so}$ prominent as at long times. The loading dependence of the overall OCFs was found to be reflected in the OCFs for different pore types at short times too.

The first order OCF can be seen to represent the evolution of the angle made by the molecular axis at time $t$ with its original orientation at $t=0$. Calling this angle $\psi$, we can thus write the first order OCF as

$$
C_{1}(t)=\langle\cos \psi\rangle
$$

The first minima in $C_{l}(t)$ signifies a change in the direction of rotation. The angle $\psi$ at that time would take the maximum value in the librational motion and that would be the angle of libration. Thus angle of libration can be obtained by taking the inverse cosine of the first minima of $C_{I}(t)$. The values of angle of libration obtained thus for different loadings and different pore types are shown in Figure 8. As expected, the sinusoidal channels have the smallest angles of libration due to severe restriction while the intersections have the largest angles of libration. The angle of libration in all pore types increase with an increase in loading. 


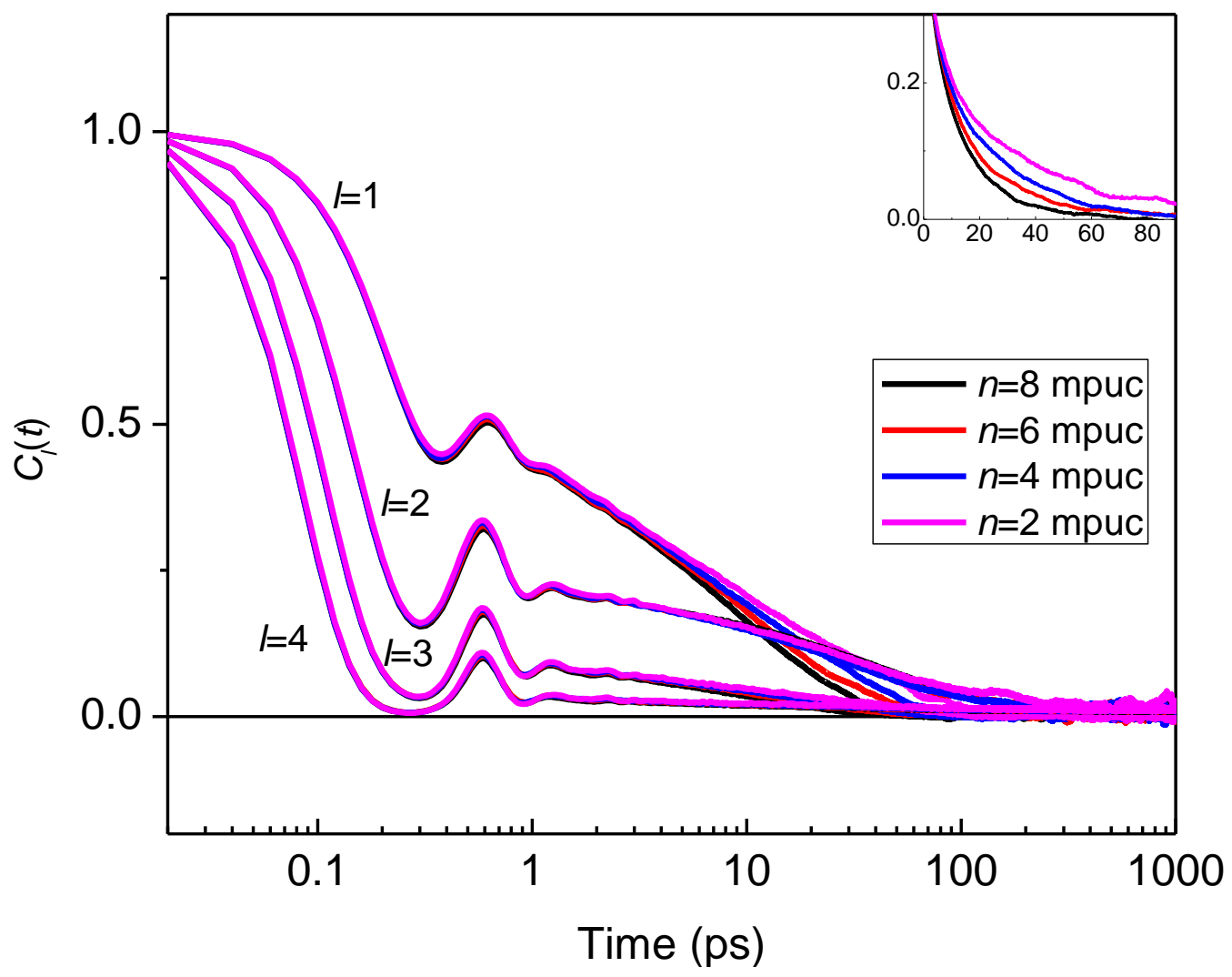

Figure 5 Orientational Correlation Functions of order 1, 2, 3 and 4, for different loadings of ethane in ZSM5. For clarity, the variation of $1^{\text {st }}$ order OCF with loading is shown in the inset. Notice that the inset is a linear scale plot with a different range from the main plot. 


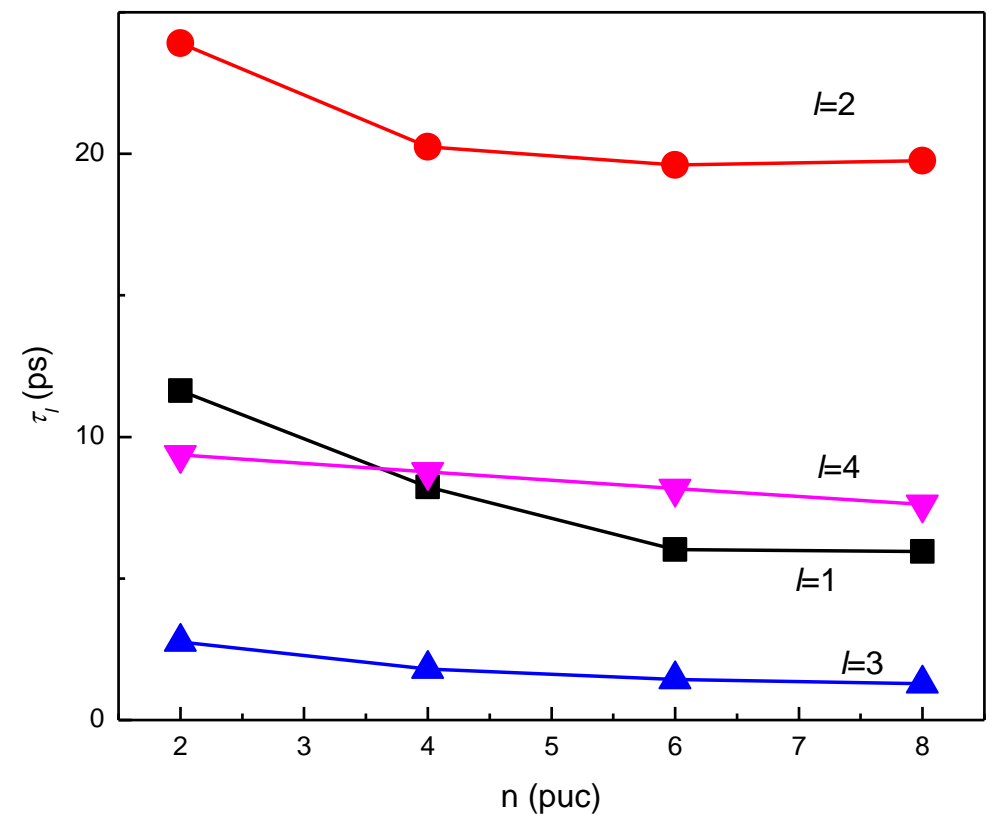

Figure 6 Orientational Correlation times obtained from the first four OCFs for different loadings of ethane in ZSM5.

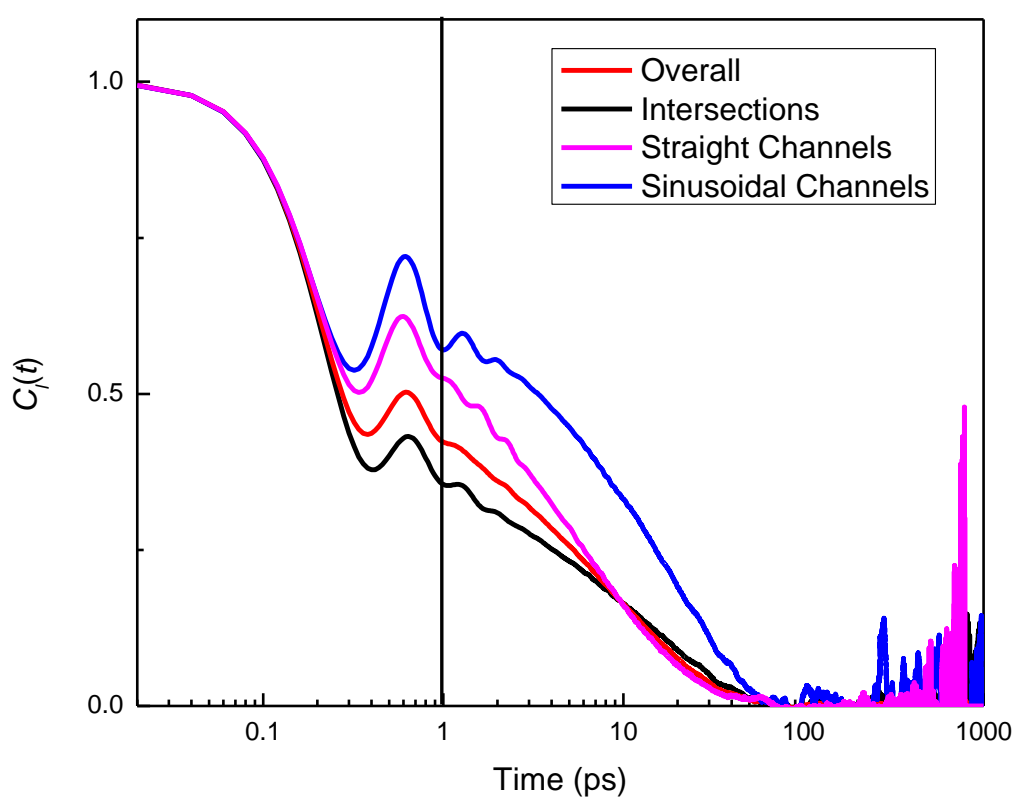

Figure 7. First order orientational correlation functions for different pore types at a loading of 8 ethane molecules per unit cell of ZSM5. The trends shown here are also observed for the OCFs of higher orders. These functions are not shown here for the purpose of clarity. 


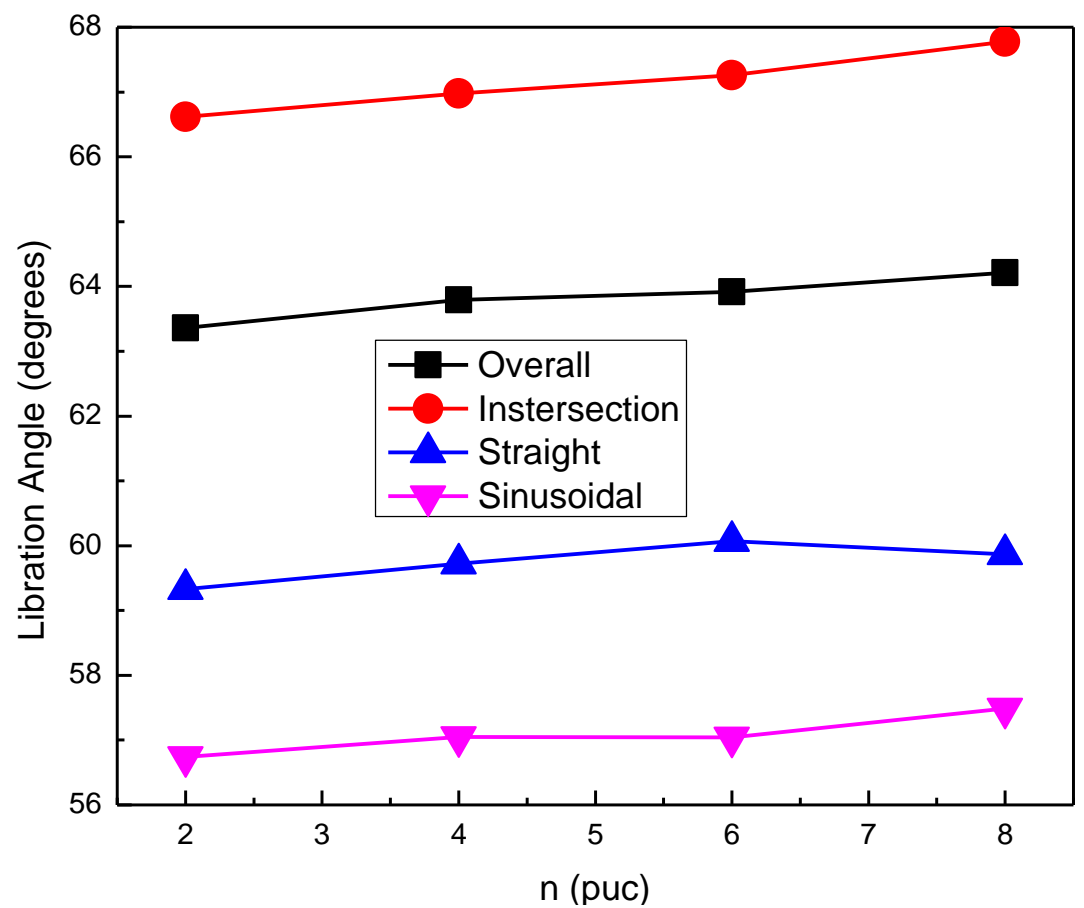

Figure 8. Angle of libration of ethane in different pores of ZSM5 as a function of loading.

\section{DISCUSSION}

The geometrical restriction imposed by the ZSM5 structure leads to anisotropy in the orientational structure and dynamics of confined ethane molecules. In a neutron scattering experiment on ethane in Na-ZSM5, Jobic et al. [22] observed that an isotropic rotational diffusion model could not satisfactorily reproduce the neutron scattering spectra. They accounted for some anisotropy in modeling their spectra by considering a continuous rotation of ethane about its molecular axis. Further, when they fit the experimental data with this uniaxial rotation model, they obtained values of radius of gyration ranging between 2 and $3 \AA$. This implied that the axis of rotation was not fixed but kept moving. This would happen in a librational motion which we have inferred from the shape of the OCFs. Thus our findings of a hindered 
orientational motion are consistent with the experimental observations. Moreover, the orientational motion getting faster at higher loadings is also consistent with the experimental findings of Jobic et al [22]. The rotational diffusion coefficient obtained by Jobic et al. for ethane in Na-ZSM5 cannot be compared with our simulation as they used a uniaxial jump diffusion model in their analysis. The mean time between successive rotational jumps they obtained (2.7 ps) has the same order of magnitude as $\tau_{3}$ obtained in our study (Figure 6).

The correlation times obtained from the first and second order OCFs in this study are smaller than those obtained for propylene in ZSM5 [21]. This is reasonable, as a smaller moment of inertia of ethane would make it faster compared to propylene. The mean angle of libration obtained for ethane in ZSM5 is also larger than that obtained for propylene. This is due to the shape of ethane molecule which allows it to trace out a larger orientational space when librating as compared to propylene. A hindrance to the orientational motion of ethane was also observed in AlPO-5 channels [27] which have a larger diameter of $1 \mathrm{~nm}$. However, the correlation times obtained for ethane in $\mathrm{AlPO}_{4}-5$ were an order of magnitude smaller than those obtained for ethane in ZSM5 implying a much faster rotation of ethane in larger pores of $\mathrm{AlPO}_{4}-5$.

For bulk fluids, molecular motion is expected to get slower at higher loadings or densities. However, under confinement, this is not always the case. For example, translational diffusion has been observed to get faster for confined propane at higher loadings in silica aerogel [28]. A similar enhancement of both translational as well as rotational motion was predicted for propane in $\mathrm{TiO}_{2}$ by $\mathrm{MD}$ simulation [29]. Chathoth et al. [30] have observed that the diffusion of methane confined in nanoporous carbon aerogel gets faster with pressure up to $27.5 \mathrm{MPa}$ after which it starts to slow down. Thus the enhancement observed in the orientational motion of ethane confined in ZSM5 with loading is consistent with the behavior under confinement with the trends 
observed in similar confined systems. The reason for this lies in the orientational space spanned by an ethane molecule in ZSM5. Our simulations show that the anisotropy in the orientational distribution of ethane molecules decreases at higher loadings. This is because at lower loadings, the ethane molecules occupy only the most favored orientations and thus the orientational distribution is highly anisotropic. As more molecules are added at higher loadings, the most favored orientations are no longer available and the newly added molecules have to take the next favored orientations. This reduces the orientational anisotropy at higher loadings. The increase in the angle of libration at higher loadings is also consistent with this behavior. As the angle of libration increases, the orientational space covered by the librational motion increases thus making the librational motion faster.

\section{CONCLUSIONS}

MD simulation studies of ethane confined in ZSM5 zeolite reveal that the orientational structure and dynamics are dependent on the location of ethane molecules in pores with different geometries. Overall, the orientational distribution of ethane in ZSM5 is anisotropic. The degree and nature of this anisotropy is different for different pore geometries. While in the pore channel intersections the anisotropy is minimal due to a relaxed spatial restriction, in the straight and sinusoidal channels, the anisotropy is greater along the $\mathrm{Y}$ and $\mathrm{X}$-directions respectively. This anisotropy is found to decrease with an increase in the loading of ethane. The orientational dynamics of ethane confined in ZSM5 indicates a hindrance to isotropic rotational diffusion. This hindrance gives rise to librational motion. The librational motion is found to be fastest in the pore channel intersections and slowest in the sinusoidal channels. This motion becomes faster 
at higher loadings consistent with the neutron scattering results on ethane in Na-ZSM5 reported elsewhere [22]. This counterintuitive loading dependence of the librational motion is explained in terms of a decrease in the anisotropy of the orientational distribution. The time scales of librational motion of ethane in ZSM5 obtained are smaller than that obtained for propylene indicating a faster libration of ethane with a smaller moment of inertia. However, the libration of ethane in ZSM5 is much slower than that in $\mathrm{AlPO}_{4}-5$ which has larger pores.

\section{Acknowledgements}

DL_POLY_4 is a molecular dynamics simulation package written by I.T. Todorov and W. Smith, and has been obtained from STFC's Daresbury Laboratory via the website http://www.ccp5.ac.uk/DL_POLY. We would also like to acknowledge computational support from the Deep Carbon Observatory cluster hosted by Rensselaer Polytechnic Institute (Peter Fox and Patrick West). SG, SP and DC receive support from the US Department of Energy, Office of Basic Sciences, Geosciences and Biosciences under grant DE-SC0006878. TL receives support from Deep Carbon Observatory.

\section{References}

[1] D. R. Cole, E. Mamontov and G. Rother in L. Liang, R. Rinaldi and H. Schoeber (Eds.)

Neutron Applications in Earth, Energy, and Environmental Sciences Springer: Berlin, 2009, pp $547-570$.

[2] D. Ruthven, Diff. Fund. 2 (2005) 77.1 
[3] J. Karger, R. Valiullin, Chem. Soc. Rev. 42 (2013) 4172.

[4] G. Buntkowsky, H. Breitzke, A. Adamczyk, F. Roelofs, T. Emmler, E. Gedat, B. Grünberg, Y. Xu, H-H. Limbach, I. Shenderovich, A. Vyalikhz and G. Findenegg, Phys. Chem. Chem. Phys. 9 (2007) 4843.

[5] K. Koga, X. C. Zheng, H. Tanaka, Chem. Phys. Lett. 285 (1998) 278.

[6] G. A. Mansoori, and S. A. Rice, Adv. Chem. Phys. 156 (2015) 197.

[7] N. Giovambattista, P. J. Rossky and P. G. Debenedetti, Phys. Rev. E 73 (2006) 041604.

[8] P. Hirunsit and P. B. Balbuena, J. Phys. Chem. C 111 (2007) 1709.

[9] A. Verdaguer, G. M. Sacha, H. Bluhm, and M. Salmeron, Chem. Rev. 106 (2006) 1478.

[10] Davide Mattia and Yuri Gogotsi, Microfluid Nanofluidics 5 (2008) 289.

[11] R. Bergman and J. Swenson, Nature 403 (2000) 283.

[12] P. K. Ghorai, S. Yashonath, P. Demontis and G. B. Suffriti, J. Am. Chem. Soc. 125 (2003) 7116.

[13] P. Demontis and G. B. Suffritti, Chem. Rev. 97 (1997) 2845.

[14] D. Chakraborty, S. Gautam, S. Mitra, A. Gil, M. A. Vicente and R. Mukhopadhyay, Chem. Phys. Lett. 426 (2006) 296.

[15] E. G. Solveyra, E. de la Llave, V. Molinero, G. J. A. A. Soler-Illia, and D. A. Scherlis, J. Phys. Chem. C 117 (2013) 3330.

[16] S. Gautam, S. Mitra, R. Mukhopadhyay and S. L. Chaplot, Phys. Rev. E 74 (2006) 041202. 
[17] R. Mukhopadhyay, A. Sayeed, S. Mitra, A.V. A. Kumar, M. N. Rao, S. Yashonath and S. L. Chaplot, Phys Rev E 66 (2002) 061201.

[18] V. K. Sharma, S. Gautam, S. Mitra, M. N. Rao, A. K. Tripathi, S. L. Chaplot and R. Mukhopadhyay, J. Phys. Chem. B 113 (2009) 8066.

[19] V. K. Sharma, S. Gautam, S.Mitra and R. Mukhopadhyay, Z. Phys. Chem. 224 (2010) 133.

[20] S. Gautam, S. Mitra, S. L. Chaplot and R. Mukhopadhyay, Phys. Rev. E 77 (2008) 061201.

[21] S. Gautam, V. K. Sharma, S. Mitra and R. Mukhopadhyay, Chem. Phys. Lett. 501 (2011) 345.

[22] H. Jobic, M. Bee, G. J. Kearly, Zeolites 12 (1992) 146.

[23] I. T. Todorov, W. Smith, K. Trachenko, M. T. Dove, J. Mater. Chem. 16 (2006)1911.

[24] H. van Koningsveld, H. van Bekkum, J. C. Jansen, Acta Cryst. B43 (1987) 127.

[25] M. G. Martin, and J. I. Siepmann, J. Phys. Chem. B 102, (1998) 2569.

[26] T. J. H. Vlugt, R. Krishna, B. Smit, J. Phys. Chem. B 103 (1999)1102.

[27] S. Bhide and S. Yashonath, J. Phys. Chem. A 106 (2002) 7130.

[28] S. Gautam, T. Liu, G. Rother, N. Jalarvo, E. Mamontov, S. Welch, J. Sheets, M. Droege and D. Cole, J. Phys. Chem. C 119 (2015) 18188.

[29] S. Gautam, and D. Cole, Chem. Phys. 458 (2015) 68.

[30] S. M. Chathoth, E. Mamontov, Y. B. Melnichenko, and M. Zamponi, Microporous Mesoporous Mat. 132 (2010) 148. 

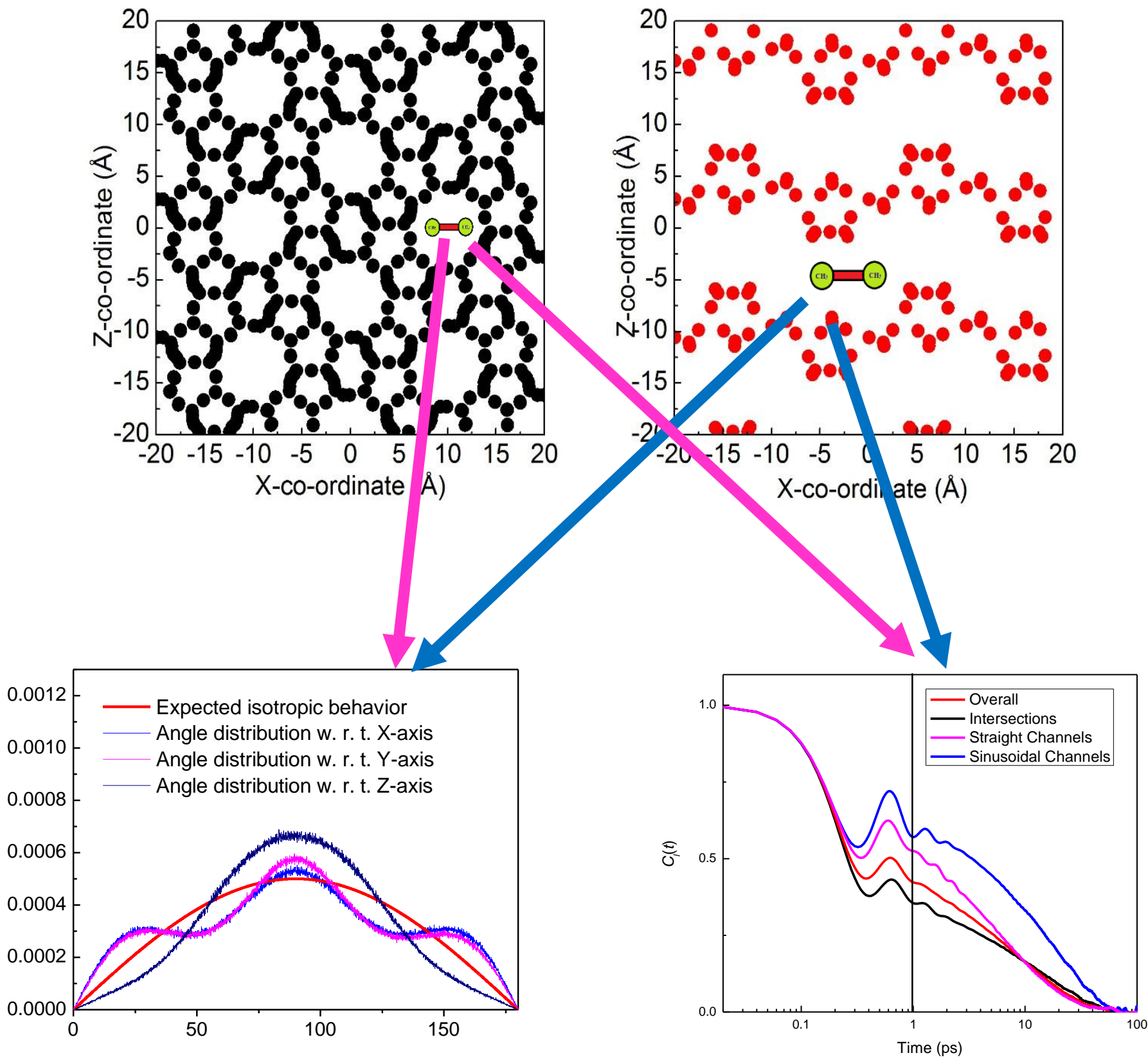